\title{
INS aided subsurface positioning for ROV surveys
}

M. van de Munt, Allseas Engineering B.V., The Netherlands

$R$ van der Velden, Allseas Engineering B.V., The Netherlands

K. Epke, Allseas Engineering B.V., The Netherlands

Topic B: subsea positioning

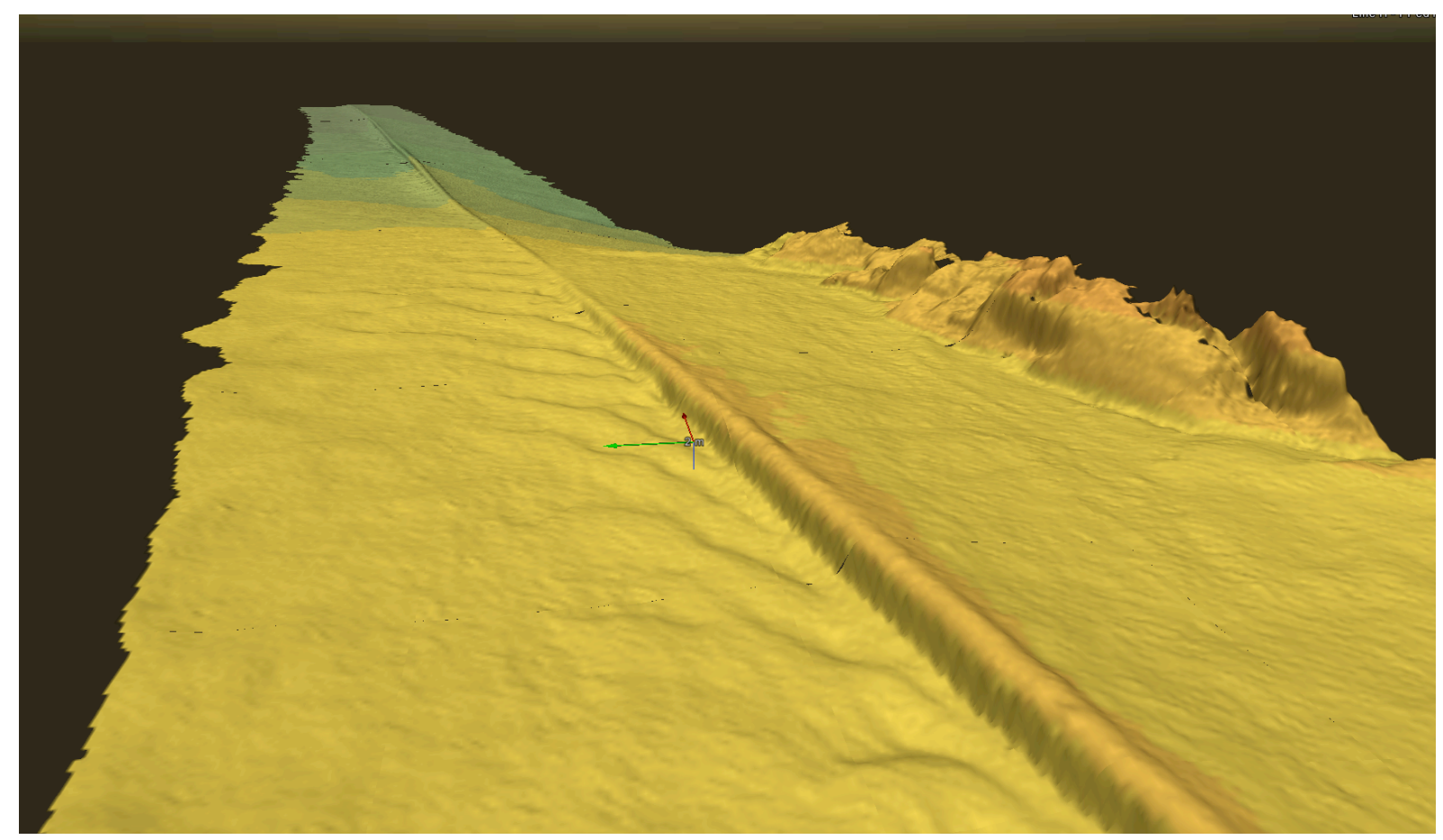

\section{INTRODUCTION}

ROV positions are established using a combination of surface and subsurface navigation systems aided by heading and motion sensors that provide global coordinates for the subsea sensor platform in near real-time. In past decades, the performance of these systems has gradually improved but not sufficiently to keep pace with the rapid move into (ultra) deep water and the related optimisation of pipeline design, especially for gas pipelines with hot mixtures. The current designs for 'hot' subsea pipelines require very tight measuring tolerances generally beyond the unaided capabilities of the acoustic (USBL) positioning systems.

A subsea pipeline which operates at temperatures and pressures above seabed ambient conditions will expand. If this expansion is restrained, for example by axial friction between the pipeline and the seabed, the axial force generated in the 'hot' pipeline could lead to localised and uncontrolled buckling. By controlling the pipeline's "relative" trajectory in three dimensions buckling can be avoided. Accurate positioning in $X, Y, Z$, is crucial for the determination of any significant small pipeline deflections. These accurate ROV pipeline surveys are referred to as out-of-straightness (OOS) surveys.

Since the early 1990s, Doppler Velocity Logs (DVLs) are installed on ROVs to enhance the Ultra Short BaseLine (USBL) position by integrating the relative short-term accuracy of the DVL with the absolute long-term accuracy of the USBL systems. Integration was based on a variety of mathematical routines of which Kalman filtering is the most commonly used. In the past decade, with the launch of commercial AUVs, the combination of INS and DVL became a primary positioning solution for these 
platforms, acquiring high precision geophysical data in deeper water. Absolute positioning of these platforms is achieved through adding sparse DGNSS or acoustics during post processing. Migration of the same technology onto the ROV platform is a challenge and is not as straightforward as it may seem.

The presentation provides the results of various trials conducted by Allseas with INS aided ROV positioning along offshore pipelines. Included are comparisons between various combinations of ROV positioning survey sensor data. Although trials were performed in both 2009 and 2012, the presentation focusses on the most recent trials with the Sonardyne SPRINT system in April 2012. The experience gained during the 2009 trials provided the basis for the recent tests.

\section{METHODOLOGY}

To analyse the performance of a ROV mounted INS, a methodology was developed to survey the same section of a pipeline on the seabed multiple times and in opposite directions.

A pipeline provides a good bench mark for the positioning systems and their repeatability, which is easily checked by comparing the processed pipeline positions for each pass. A ROV mounted multibeam is required to generate the high density data necessary to accurately detect the top of the pipeline. This then allows for an accurate comparison between the pipeline positions derived from the various passes.

To check the impact of the range dependent USBL positioning accuracies in the equation, it is best to repeat the test for different water depths, covering both shallow and deep water.

The integration of USBL, DVL and INS was first adopted by Allseas for a project in Northwest of Australia. INS/DVL in combination with a conventional acoustic positioning system had to demonstrate the ability to detect a $20 \mathrm{~cm}$ deflection over a $50 \mathrm{~m}$ length of pipeline. Tests were performed with the Kongsberg HAIN system and IXSEA PHINS. Two years later, a similar set-up was tested with a Sonardyne SPRINT/Lodestar system offshore the Shetland Islands.

\section{HARDWARE AND SOFTWARE}

For both tests, offshore NW Australia and the Shetlands, an Allseas Schilling Robotics UHD ROV system was used as the instrument carrier. This system has a patented station keeping facility, allowing for steady and automatically controlled guidance along a predefined route section.

\begin{tabular}{|l|l|}
\hline \multicolumn{2}{|c|}{ Standard survey equipment fitted on the ROV in addition to INS } \\
\hline \multicolumn{1}{|c|}{ Instrument } & \multicolumn{1}{c|}{ Type } \\
\hline Surface positioning & Veripos Ultra \\
\hline Vessel heading and motion sensor & IXSEA Octans \\
\hline Underwater positioning & Kongsberg Dual HiPAP500 \\
\hline Transponders & Sonardyne WSM \\
\hline ROV heading and motion sensor & IXSEA Octans \\
\hline Multibeam echosounder & Dual-head Reson Seabat 7125 \\
\hline Depth sensor & Paroscientific Digiquartz \\
\hline Altimeter & Kongsberg 1007 \\
\hline Doppler Velocity Log & RDI 1200kHz Workhorse Navigator \\
\hline Sound velocity sensor and CTD probe & Valeport SVX2 \\
\hline Obstacle avoidance sonar & Kongsberg MS1000 \\
\hline
\end{tabular}




\begin{tabular}{|l|l|}
\hline \multicolumn{2}{|c|}{ Survey software in addition to INS software } \\
\hline \multicolumn{1}{|c|}{ Package } & \multicolumn{1}{|c|}{ Type } \\
\hline Online DGNSS QC & Veripos VeriFy QC \\
\hline Online positioning & EIVA NaviPaC \\
\hline ROV sensor data logging & EIVA NaviScan \\
\hline Survey data processing & EIVA NaviEdit \& NaviModel 3D \\
\hline
\end{tabular}

\begin{tabular}{|l|l|}
\hline \multicolumn{2}{|c|}{ ROV INS hardware \& software } \\
\hline \multicolumn{1}{|c|}{ Manufacturer } & Hardware - (processing) software \\
\hline IXSEA & PHINS - Delph-INS \\
\hline Kongsberg & ROV HAIN - NavLab \\
\hline Sonardyne & Lodestar SPRINT - Janus \\
\hline
\end{tabular}

\section{INSTALLATION}

Two types of installation were trialled. In 2009 a bracket was made at the front of an UHD ROV to fit the integrated IMU and DVL. In 2012 the instruments were separated: the INS was mounted on the survey skid whereas the dedicated DVL and depth sensor were mounted at an offset.

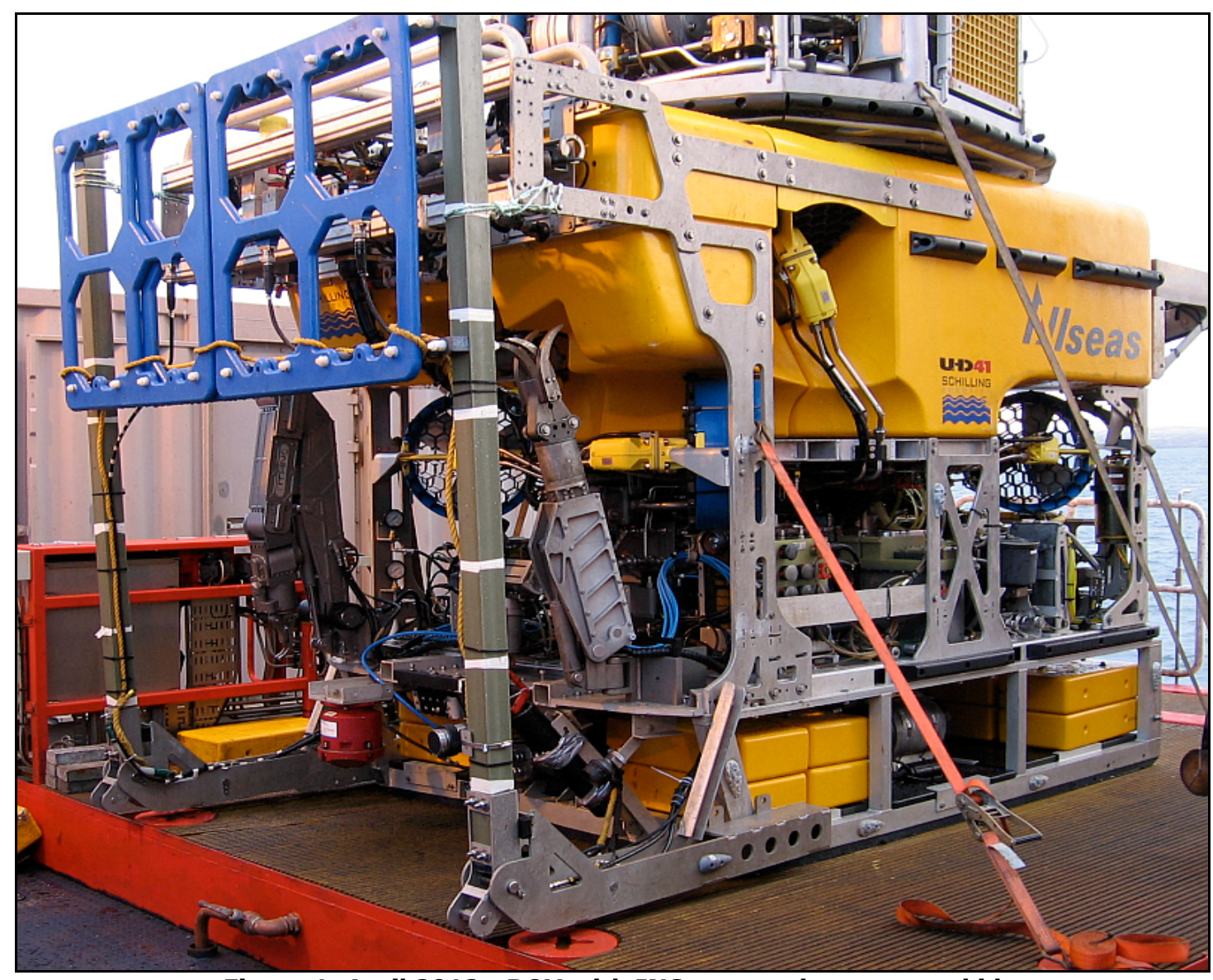

Figure 1: April 2012 - ROV with INS mounted on survey skid 
The required calibrations were performed according to standard procedures. The dedicated DVL was calibrated to align with the heading sensor in the INS. For clarity the calibration sequence is listed in the table below.

\begin{tabular}{|l|}
\hline \multicolumn{1}{|c|}{ Calibrations prior to ROV INS survey work } \\
\hline \multicolumn{1}{|c|}{ Task } \\
\hline Local vessel and ROV offsets \\
\hline Calibration SV and CTD probe \\
\hline DGNSS health check \\
\hline Vessel gyro, pitch, roll calibration \\
\hline ROV gyro, pitch, roll calibration \\
\hline APOS (HiPAP) calibration \\
\hline ROV dual head multibeam calibration \\
\hline DVL alignment to INS \\
\hline Position confirmation checks \\
\hline
\end{tabular}

\section{RESULTS}

\section{Test}

In 2009 ROV INS tests were performed for a project in NW Australia. A detection criterion of $20 \mathrm{~cm}$ deflection over a $50 \mathrm{~m}$ length was defined. Both IXSEA and Kongsberg INS solutions were tested. Minimum four ROV passes along same pipeline segments were run in shallow and deep water depths of $85 \mathrm{~m}$ and $827 \mathrm{~m}$ respectively. A dual headed multibeam mounted on the ROV provided detailed digital terrain models along the pipeline. Calculated values for the top of the pipeline were extracted from digital terrain models. Results were compared to check compliance with the above described deflection criteria.

The IXSEA product provided a solution in real-time from a PHINS. However, the results at the time did not meet the criteria. After further detailed study in the office, post-processed results could be provided ultimately that passed the test.

The Kongsberg all integrated system provided on board post-processed results above expectations and within a short time frame.

Subsequently, the Kongsberg solution was selected for the out-of-straightness survey works.

\section{Test}

Based on the experience in 2009, a further test was scheduled to also investigate the performance of the new Sonardyne Lodestar SPRINT system and to compare different positioning solutions with and without INS, both in real-time and post-processed.

The test was performed in April 2012, with a similar type UHD ROV system equipped with the same standard survey instrumentation as in 2009.

The Sonardyne Lodestar INS, was configured to provide both an INS aided positioning solution and heading, pitch, roll and heave data.

$500 \mathrm{~m}$ segments of pipeline were surveyed four times in opposite directions. For the first segment, which is highlighted in this paper, lines $A-D$, lines $A$ and $C$ are surveyed in opposite direction to lines $B$ and $\mathrm{D}$. The water depth at the survey location varied between $60 \mathrm{~m}$ and $80 \mathrm{~m}$. 
The collected data was processed using four different positioning solutions:

1. Post-processed DVL enhanced USBL positioning, using EIVA NaviEdit software;

2. Post-processed DVL enhanced USBL positioning, without INS, further smoothed using in-house developed filter based on pipeline characteristics based on minimum bending radii;

3. Real-time INS aided by USBL/DVL positioning, using Sonardyne SPRINT Kalman filtered output;

4. Post-processed INS aided by USBL/DVL positioning, using Janus post-processing software.

Each positioning solution was used to generate detailed digital terrain models. Examples from the resulting DTMs using the USBL/DVL and the post-processed INS solution are shown in Figure 2.

The USBL/DVL solution (1) showed artefacts in both the horizontal and vertical position of the pipeline. These are caused by USBL uncertainties and swell effects recorded by the depth sensor. Of all four positioning solutions, the post-processed INS (4) showed the best result.

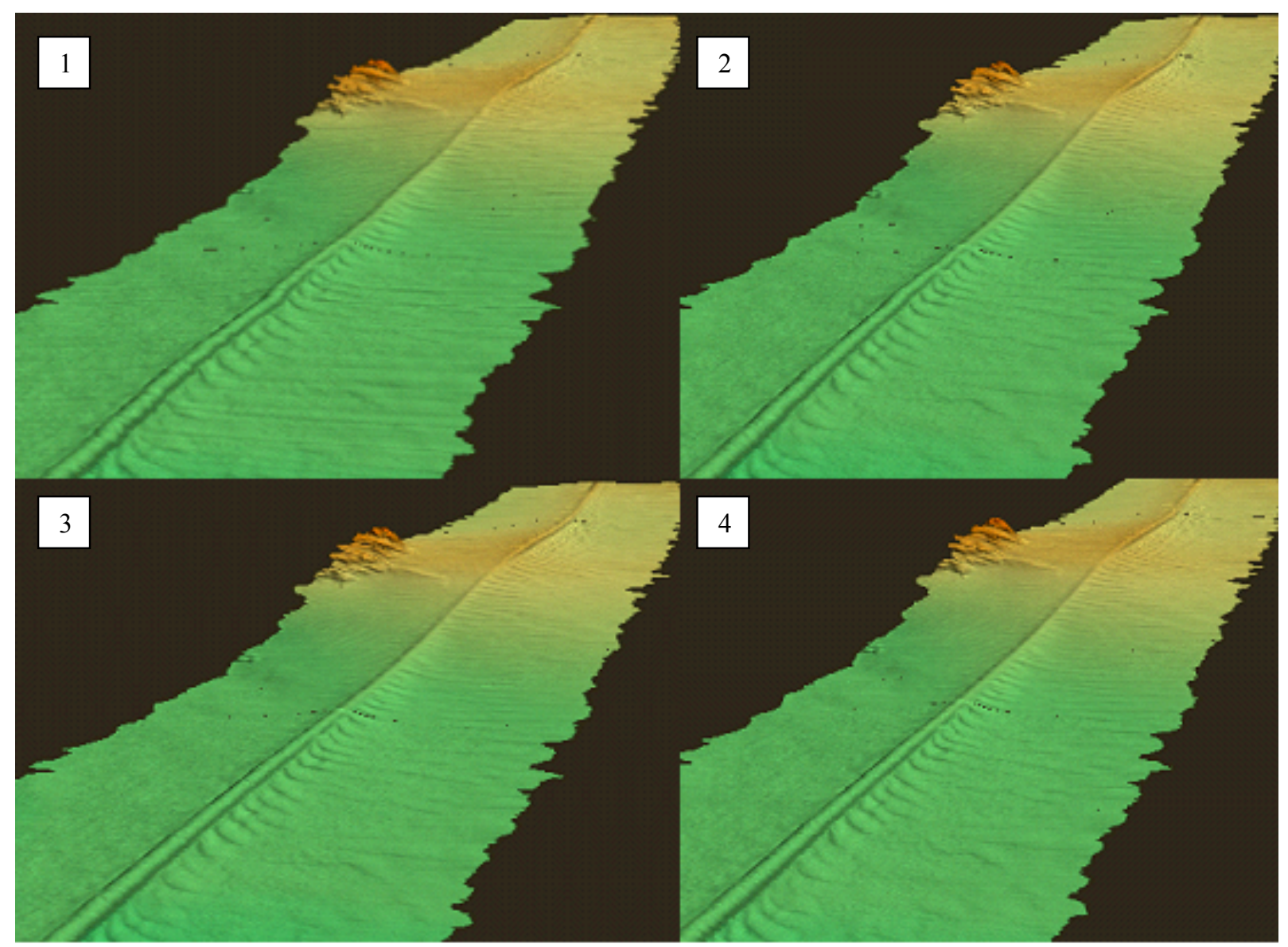

Figure 2: Resulting DTMs for the four positioning solutions - EIVA Navimodel

The DTMs were used to determine the position of the pipeline. Using these pipeline positions, distance-of-track (DOT) offsets were computed relative to a reference line. The offsets allowed for a relative comparison in the $2 \mathrm{D}$ horizontal plane, as shown in figure 3. 


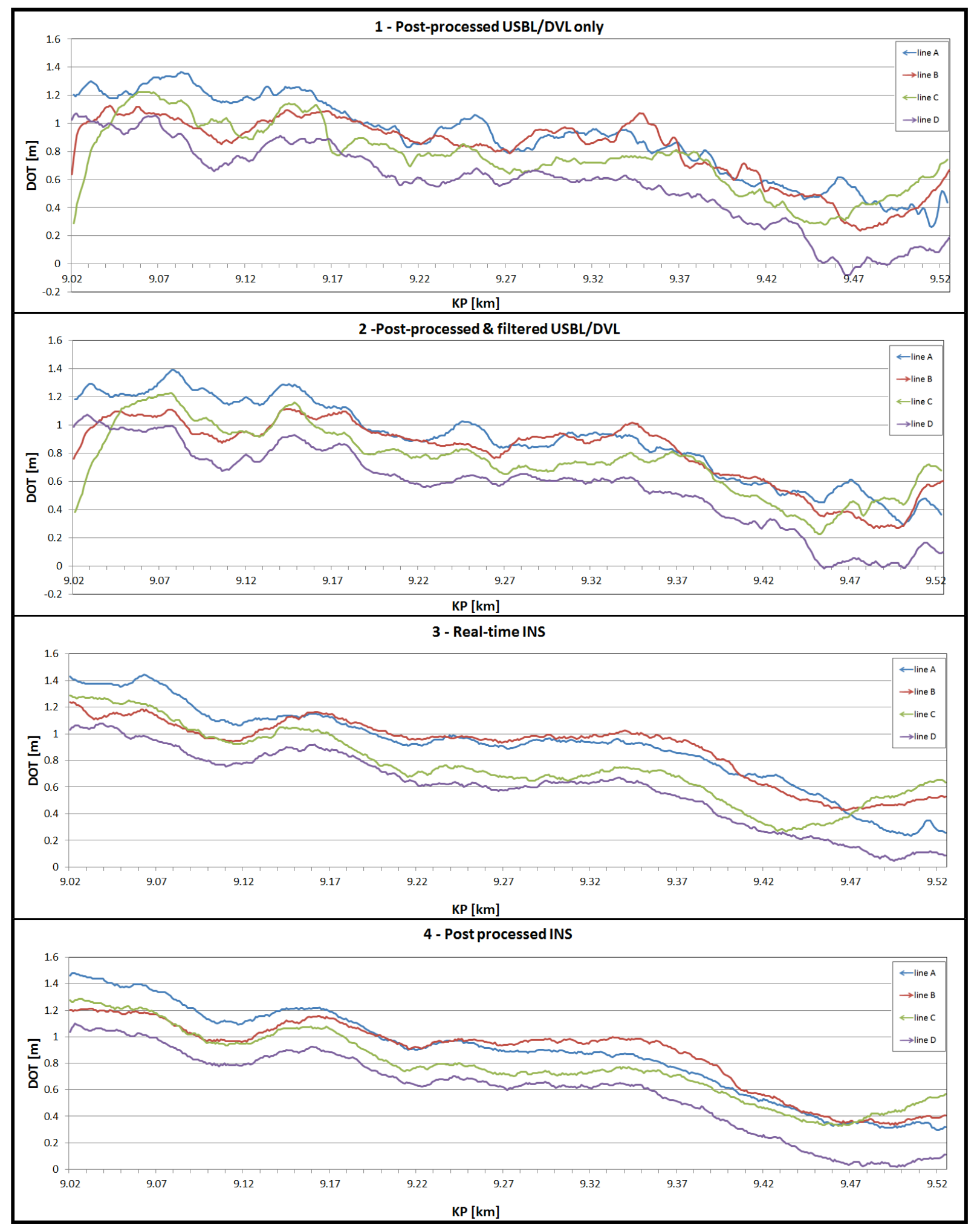

Figure 3: Relative comparison based on DOT values 
Comparing the four graphs of figure 3, correlation between the four survey lines seemed to improve from positioning solution 1 to positioning solution 4 .

This is better shown in figure 4, which provides a better visualisation on the detection capability of lateral pipeline deflections for each positioning solution.

Results of figure 4 were computed using the following method:

- For each positioning solution, the initial DOT values were set to zero;

- The maximum difference between the DOT values for each line were calculated at a metre interval up to a distance of $100 \mathrm{~m}$;

- This process was repeated by moving the start point for the $100 \mathrm{~m}$ section one metre along the data set of $500 \mathrm{~m}$;

- Each point with a metre interval now included multiple values for the maximum DOT differences;

- By calculating the average and standard deviation for the DOT differences at a metre interval, the uncertainty for the measurements was determined within a $95 \%$ confidence level.

In figure 4 , the maximum uncertainty in the measurements is shown for each metre distance over a length of $100 \mathrm{~m}$. Based on a $95 \%$ confidence level for solution 4, a lateral pipeline deflection of $20 \mathrm{~cm}$ can be determined over travelled distances of up to $100 \mathrm{~m}$.

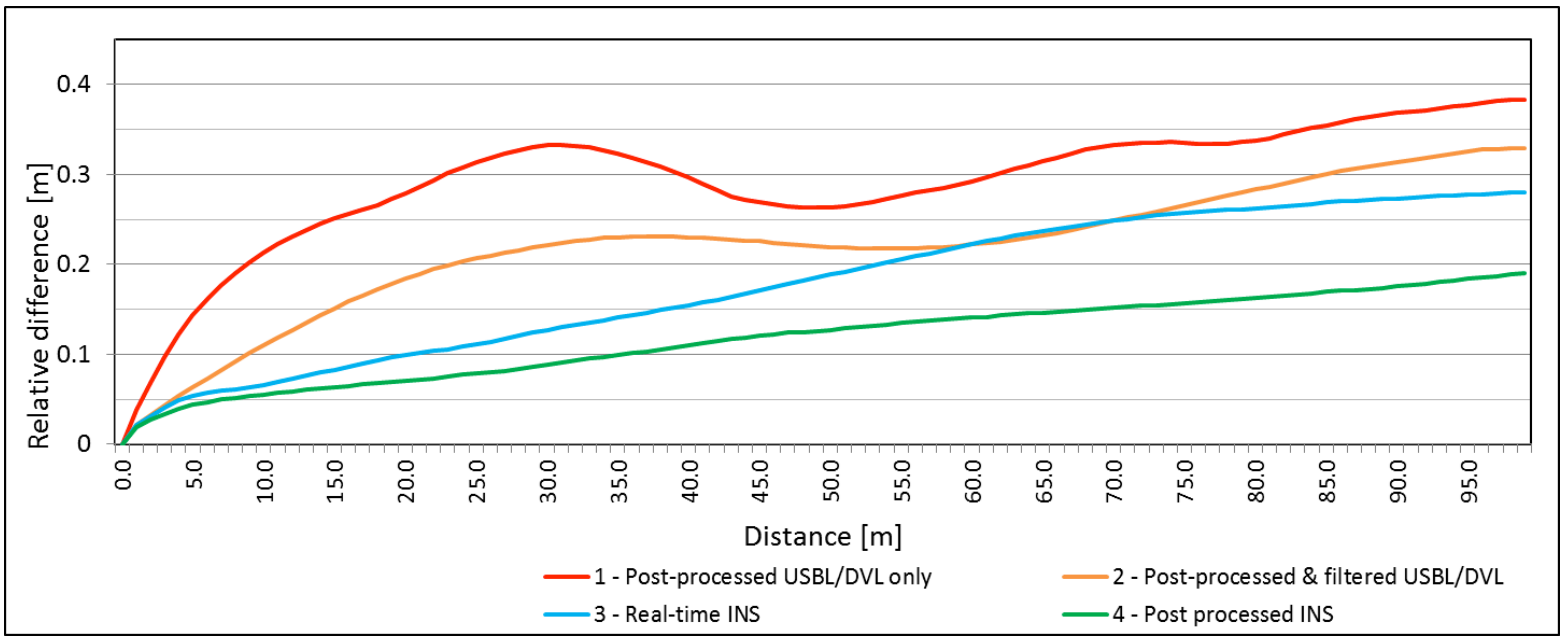

Figure 4: Relative uncertainty over distance for the different positioning solutions

\section{CONCLUSIONS}

2009 Test

The test with the IXSEA system showed at the time that a real-time (PHINS) INS aided by USBL/DVL positioning solution could not meet the specifications.

The Kongsberg system clearly demonstrated that on board post-processed HAIN INS aided by HiPAP/DVL positioning in a water depth ranging from $85 \mathrm{~m}$ to $827 \mathrm{~m}$ was accurate with good correlation between subsequent survey runs along the same pipeline segment. In the detailed digital terrain models along the same pipeline segment, the positions of anodes, field joints, as well the curvature of the pipeline was checked. This test in NW Australia demonstrated that an integrated (HAIN) ROV INS is capable of detecting a $20 \mathrm{~cm}$ pipeline deflection over a $50 \mathrm{~m}$ pipeline length after on board post-processing.

2012 Test 
Analyses of the April 2012 results showed as expected that the post-processed INS provided the best positioning solution. Results exceeded the out-of-straightness survey specifications to detect a deflection of $20 \mathrm{~cm}$ over a $50 \mathrm{~m}$ pipeline length for water depths ranging from $60 \mathrm{~m}$ to $80 \mathrm{~m}$.

The fact that the IMU was not mounted above the DVL, was not observed to have a significant impact on the results.

Furthermore, the trials demonstrated that integration of different manufacturer type survey instrumentation, such as Kongsberg HiPAP, RDI DVL and Paroscientific Digiquartz, provided good results facilitating universal deployment of the methodology. 